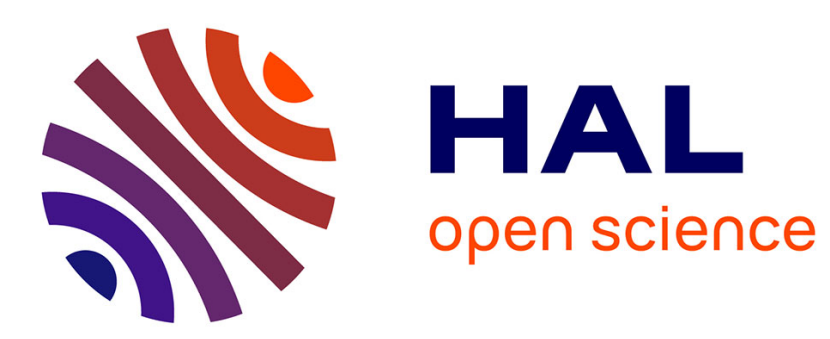

\title{
Hypermédias et éducation : des repères
}

Eric Bruillard, Brigitte de La Passardière

\section{To cite this version:}

Eric Bruillard, Brigitte de La Passardière. Hypermédias et éducation: des repères. Sciences et Techniques Educatives, 1994, 1 (1), pp.17-37. edutice-00109582

HAL Id: edutice-00109582

https://edutice.archives-ouvertes.fr/edutice-00109582

Submitted on 24 Oct 2006

HAL is a multi-disciplinary open access archive for the deposit and dissemination of scientific research documents, whether they are published or not. The documents may come from teaching and research institutions in France or abroad, or from public or private research centers.
L'archive ouverte pluridisciplinaire HAL, est destinée au dépôt et à la diffusion de documents scientifiques de niveau recherche, publiés ou non, émanant des établissements d'enseignement et de recherche français ou étrangers, des laboratoires publics ou privés. 
Hypermédias et éducation : des repères

Eric Bruillard — Brigitte de La Passardière*

IUFM de Créteil

Route de Brévannes

94388 Bonneuil cedex

* Université Pierre et Marie Curie - Paris VI

Laboratoire MASI

4 place Jussieu, 75252 Paris cedex 05

RESUME : Cet article dresse un rapide panorama des applications éducatives actuelles des hypertextes et des hypermédias. D'une part, nous abordons les usages possibles dans l'éducation et d'autre part, nous analysons les concepts liés aux recherches en cours. En effet, le domaine des hypertextes ayant connu une évolution extrêmement rapide, ses contours sont encore mal définis. On constate ainsi de nombreuses confusions entre les notions de multimédias et d'hypermédias. Il nous semble donc utile de préciser ce que recouvre le concept d'hypertexte et de passer en revue les problèmes associés à la navigation. Du côté des usages, il s'agit de décrypter une situation relativement complexe et d'établir une classification, même imparfaite, qui permet de mieux situer les différents possibles. La première partie de cet article s'intéresse donc à l'hypertexte et à la navigation en général, sans connexion particulière avec l'éducation. La seconde partie est, quant à elle, centrée sur les applications éducatives.

ABSTRACT : This paper makes a quick survey of the present educative applications in hypertext and hypermedia. Fisrt, we study possible uses in education. Then, we analyse concepts associated with present research. Hypertext domain is very new. So contours are fuzzy. There are many confusions between hypermedia and multimedia notions. We believe it is useful to precise what is hypertext and review navigation tools. Regarding uses, we try to establish a classification in order to situate possibilities. In the first part of this paper, we present hypertext in general. The second one is aimed at educational point of view.

MOTS-CLES : hypertexte, hypermédia, navigation, informatique éducative, usages.

KEY WORDS : hypertext,hypermedia, browsing, navigation tools, educational software, uses.

\section{Introduction}


Cet article dresse un rapide panorama des applications éducatives actuelles des hypertextes et des hypermédias. Notre objectif est de confronter deux aspects, celui des usages possibles dans l'éducation, c'est-à-dire des différentes façons dont les acteurs du terrain s'approprient les hypermédias et celui des recherches dans le domaine des hypertextes, c'està-dire des concepts sur lesquels travaillent les chercheurs. En effet, le domaine des hypertextes ayant connu une évolution extrêmement rapide ces dernières années (le premier colloque sur ce thème s'est tenu en 1987 en Caroline du Nord), ses contours sont encore mal connus. On constate ainsi de nombreuses confusions entre les notions de multimédias et d'hypermédias, entretenues en partie par les annonces publicitaires. S'entendre sur des définitions, en accord avec celles couramment admises, est important pour éviter de creuser un fossé trop important entre la recherche et les applications éducatives. En particulier, il nous semble utile de préciser ce que recouvre le concept d'hypertexte et de passer en revue les problèmes associés à la navigation. Du côté des usages, il s'agit de décrypter une situation relativement complexe et d'établir une classification, même imparfaite, qui permet de mieux situer les différents possibles.

La première partie de cet article s'intéresse donc à l'hypertexte et à la navigation en général, sans connexion particulière avec l'éducation. La seconde partie est, quant à elle, centrée sur les applications éducatives.

\section{Notions générales sur les hypertextes}

\subsection{Qu'est-ce qu'un hypertexte?}

Quand on cherche des définitions précises des hypertextes, on s'aperçoit que les avis des auteurs divergent fortement et que le seul point d'accord est qu'il n'existe pas de théorie unifiée des systèmes hypertextes, ni même de définition vraiment satisfaisante de ce qu'est un système hypertexte. Marc Nanard observe que le concept lui-même ne s'est pas encore stabilisé, qu'il s'agit avant tout de chercher des moyens pour rendre de l'information plus facilement accessible à l'homme et que «toutes les réalisations s'appuient sur des techniques existantes, cherchent à les unifier et acceptent de remettre en cause les contraintes qui sont inacceptables pour un usager libre et qui n'étaient là que pour l'élégance d'une théorie ou pour le confort du programmeur» [Nanard 90].

La courte histoire de l'hypertexte est relatée dans de nombreux ouvrages [Shneiderman \& Kearsley 89, Nielsen 90, Berk \& Devlin 91, Nyce \& Kahn 91, Laufer \& Scavetta 92]. De multiples liens la relient à l'émergence d'une technologie visant à fournir des outils cognitifs 
à l'homme, à mettre en place de nouvelles formes de travail et de pensée associant étroitement l'homme et la machine : la «pensée assistée par ordinateur» [Rheingold 93]. Cette histoire est d'abord celle de visionnaires : Bush, Engelbart, Nelson, décrivant et poursuivant des projets très ambitieux, dont les objectifs déclarés sont d'étendre les capacités intellectuelles humaines avec l'assistance des machines. Leurs idées nourrissent encore des recherches qui, depuis une dizaine d'années, aboutissent à de multiples réalisations et applications, montrant un domaine en pleine expansion.

L'hypertexte est, somme toute, un concept émergent. Meyrowitz, se basant sur le constat que deux individus ne peuvent s'entendre sur les termes d'hypertexte et d'hypermédia, propose de rebaptiser la recherche sur ce thème "responsive document research", autrement dit «recherche sur les documents réactifs» [Meyrowitz 89, Meyrowitz 90]. Cette nouvelle dénomination a l'avantage de souligner l'aspect essentiel qui est de savoir à qui et pourquoi les documents doivent réagir. Meyrowitz représente ce champ de recherche comme une roue ayant de nombreux rayons : les outils de construction de logiciels graphiques (HyperCard, SuperCard, Toolbook), les tables d'orientation ou "browser" pour la documentation technique (Document Examiner, Concordia), les gestionnaires de documents multimédias basés sur le temps (MacroMind's Director), les gestionnaires d'idées (Outline Processors, More, Guide, NLS), les environnements hypermédias intégrés (NoteCards, Guide, Intermedia, Xanadu), les systèmes de représentation des connaissances et de support d'argumentation basés sur l'hypertexte (g-IBIS), les systèmes de recherche documentaire...

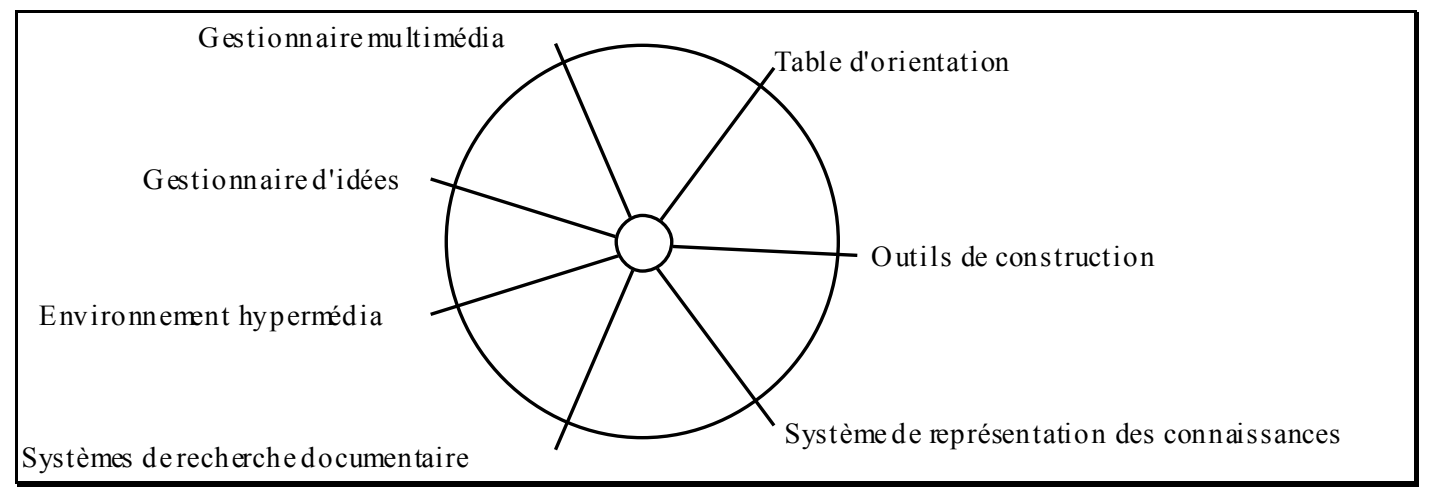

Figure 1 : champs de recherche liés aux hypertextes

Cette énumération, non exhaustive, montre que la simple connection de noeuds par des liens n'est pas le coeur de l'hypertexte. Il s'agit plutôt d'intégrer de nombreuses fonctionnalités permettant de rendre les documents réellement réactifs (responsive) en utilisant des systèmes de gestion d'idées (outlining), des liens, de la recherche de mots, des présentations multimédias synchronisées, des inférences dans des bases de connaissances. 
Les systèmes disponibles à l'heure actuelle sont peu satisfaisants, puisqu'ils s'intègrent encore imparfaitement à l'environnement de travail quotidien, et n'arrivent pas à se fondre dans les autres logiciels utilisés [Meyrowitz 89]. En fait, la recherche sur les hypertextes se trouve au croisement des recherches sur les bases de données, sur les systèmes à bases de connaissances et sur les systèmes documentaires. Au delà des diverses implémentations informatiques et de leurs caractéristiques spécifiques, les hypertextes sont des dispositifs conçus pour permettre à l'homme de traiter des ensembles d'informations. En ce sens, «un hypertexte peut être vu comme une base de données distribuée d'îlots d'information, avec des références croisées utilisant différents types de liens, accessible à un grand nombre de personnes (usagers ou auteurs) et permettant différentes activités de navigation, de manipulation, de communication et de coopération» [Maurer 92].

\subsection{Caractéristiques des systèmes existants}

En général, l'hypertexte se présente comme un dispositif informatisé permettant l'interconnexion de documents de divers types, ceci non sur un modèle hiérarchique ou relationnel, mais par des mécanismes associatifs sous contrôle de l'utilisateur [Shneiderman \& Kearsley 89, Nielsen 90, Berk \& Devlin 91]. L'utilisation ou la lecture d'un hypertexte s'effectue de manière non séquentielle et multi-dimensionnelle ; les documents ainsi organisés sont destinés à être lus et éventuellement complétés suivant différents chemins parcourus au gré de l'utilisateur.

Compte tenu des applications les plus courantes, un hypertexte est souvent représenté par un réseau de noeuds et de liens, dans lequel les noeuds sont des documents (ou ressources) et les liens, des références entre les noeuds. Dans ce contexte, l'utilisateur est avant tout un lecteur, mais un lecteur actif.

On appelle «navigation» le déplacement à travers ce réseau, métaphore spatiale et intentionnelle qui souligne bien le rôle actif du lecteur. Elle s'effectue par l'activation de points d'ancrage correspondant aux origines des liens qui sont habituellement repérables par une typographie différente (caractères en gras, en italique, dans une autre police...), par une icône, ou encore par un marquage caractéristique [Utting \& Yankelovich 89]. Leur activation a pour effet de présenter au lecteur le contenu du noeud destination. Ainsi, le lecteur choisit l'option qu'il va suivre au fur et à mesure de sa lecture. C'est donc bien lui et non l'auteur de l'hypertexte, qui trace son chemin dans la «mer d'informations». 
Le concept d'hypertexte, qui correspond d'une manière assez générale à la possibilité d'établir des liens ou de naviguer à travers des ensembles de documents, recouvre en fait deux notions complémentaires :

- l'intégration, grâce à laquelle le système est capable de piloter des ressources de nature différente, éventuellement produites et gérées par d'autres applications (en ce sens, les hypertextes interviennent dans les techniques d'interfaçage) ;

- une organisation, favorisant des accès associatifs aux informations.

Ainsi, le concept d'hypertexte recouvre à la fois un aspect d'organisation interne (les liens et les noeuds) et un aspect de manipulation externe (les points d'ancrage et la navigation). Néanmoins, les systèmes que l'on pilote par pointage de zones particulières ou d'icônes ne sont pas à proprement parler des hypertextes, mais plutôt des systèmes que l'on qualifiera de «systèmes à base de navigation». Cette possibilité se retrouve dans de nombreuses applications informatiques, et notamment dans les interfaces graphiques des systèmes d'exploitation sans pour autant rassembler l'ensemble des caractéristiques des hypertextes (et notamment l'absence de liens directs entre les ressources elles-même).

Le terme hypermédia est souvent employé pour désigner un hypertexte dans lequel les noeuds ne sont pas uniquement des documents textuels mais peuvent correspondre à d'autres médias comme des images, de la vidéo, du son... Cependant, l'aspect multimédia n'est pas en soi suffisant pour justifier l'appelation hypermédia.

Dans la suite, on utilisera le terme générique d'hypertexte ${ }^{1}$ pour désigner aussi bien des systèmes limités au texte que des systèmes intégrant d'autres médias comme les images animées ou le son. Cette dénomination a l'avantage de souligner la spécificité du concept hypertexte dans des environnements mono ou multi-médias.

\subsection{Navigation}

La navigation est une des caractéristiques importantes des hypertextes. Elle offre à l'utilisateur une grande liberté d'exploration, tout en préservant une réelle simplicité de mise en oeuvre. Cependant, si le système se charge de trouver la destination des liens activés, c'est à l'utilisateur qu'incombe la responsabilité de donner $d u$ sens au chemin qu'il construit par ses choix successifs. Il est alors confronté à des problèmes d'orientation principalement dus, à l'ignorance de la structure du réseau et de la position courante, à la gestion de la tâche

1 On trouve aussi le terme "hyperdocument", qui est utilisé plutôt dans un contexte de recherche documentaire [Balpe 91] 
rendue complexe par l'absence de stratégie cohérente de parcours et de trop nombreuses digressions [Foss 88]. Ceci entraîne une surcharge cognitive liée à l'effort et à la concentration nécessaires pour maintenir différents travaux ou chemins en même temps, et au manque d'indices discursifs qui sont généralement présents dans les textes traditionnels.

Pour tenter de pallier à ces difficultés, diverses aides à la navigation sont intégrées dans les systèmes. Ces aides fournissent des repères aux utilisateurs et doivent leur permettre de répondre à des questions telles que : où suis-je ? qu'est-ce que je peux faire maintenant ? qu'est-ce qui est important ? qu'est-ce que je n'ai pas encore vu ? comment puis-je m'y rendre? [Conklin 87]. Ces aides, que l'on peut qualifier d'outils en tant qu'éléments actifs de l'interface, permettent notamment d'avoir une idée des sujets traités dans la base d'informations, de s'orienter, ou encore de choisir un chemin d'accès vers l'information recherchée [Shneiderman \& Kearsley 90]. On les répartit généralement en trois catégories : les aides ponctuelles, les aides spatiales et structurelles et les aides historiques [Nielsen 90a, Utting \& Yankelovich 89, Vatonne 90].

\section{Aides ponctuelles}

Les aides ponctuelles permettent de mieux comprendre le contenu du noeud courant et de préciser, d'une manière locale, les pistes intéressantes à explorer. Elles sont généralement présentes dans chacun des noeuds du réseau. Par exemple, avec Hyperties, il est possible d'obtenir un bref résumé de la destination d'un lien [Shneiderman \& Kearsley 90]. Dans d'autres systèmes, on peut choisir les modes de visualisation des points d'ancrage [Bruillard 91a].

\section{Aides spatiales et structurelles}

Ces outils fournissent des informations sur le contenu de la base et son organisation. Ils aident l'utilisateur à s'orienter dans le réseau d'informations ${ }^{2}$, spécialement si celui-ci est vaste et complexe, et lui permettent de savoir où il est et comment il peut atteindre un noeud particulier.

- Les vues générales (overview maps) montrent l'organisation de la base à un niveau «macroscopique». Elles rendent compte des liens existants entre les différents noeuds ou groupes de noeuds de la base. Elles sont comparables à des tables d'orientation; elles sont appelées cartes globales (global maps) dans Intermedia [Meyrowitz 86, Utting \&

2 Le réseau d'informations est aussi appelé "hyper-espace" 
Yankelovich 89] et désignées sous le terme de diagrammes structurels (browsers) dans NoteCard [Halasz 88]. En fait, elles ne sont réellement utiles que si l'hyper-espace n'est pas trop grand. Dans le cas contraire en effet, elles deviennent vite illisibles en raison du nombre important de liens à représenter sur l'écran, généralement de petite taille.

- Les vues locales (local maps) présentent l'environnement du point courant, c'est-à-dire du noeud sélectionné par l'utilisateur. Elles montrent, en fait, tous les documents liés à ce point [Garrett 86, Garg 88, Utting \& Yankelovich 89].

- Les vues grands angles (fisheyes) peuvent être assimilés à des vues locales dans la mesure où elles rendent compte de l'environnement contextuel du point courant. Comme l'objectif photographique du même nom, elles grossissent et détaillent les objets proches, et diminuent et estompent les objets éloignés [Furnas 86].

- Les filtres (filters) sont utilisés pour améliorer la lisibilité des vues en réduisant le nombre de liens à afficher. Leur utilisation nécessite un typage préalable des liens ou des noeuds. Dans NLS/Augment, par exemple, il est possible de couper une structure hiérarchique à n'importe quel niveau ou bien de préciser le nombre d'objets à afficher à chaque niveau. Il est aussi possible de spécifier ses propres filtres en précisant les critères d'affichage des noeuds et liens [Engelbart \& English 68].

- Les visites guidées (guided tours) correspondent à des chemins prédéfinis qui permettent à l'utilisateur de parcourir une partie signifiante du réseau.

- Les index (indexes) reflétent la structure de la base. Ils sont particulièrement bien adaptés aux organisations hiérarchiques. Dans TextNet, des noeuds particuliers (appelés tocs) servent à définir la structure de l'information des noeuds textuels (appelés trunks) [Trigg 83]. Cette facilité est aussi présente dans NoteCards, où il est possible de structurer un vaste ensemble de cartes (appelées notecards) via des cartes spéciales (appelées fileboxes). La structure sous-jacente est alors visualisée par un afficheur graphique [Halasz 88]. De même, dans g-IBIS, la principale tâche de l'afficheur graphique est le calcul des vues locales, c'està-dire la définition de l'environnement du point courant à partir de la structure hiérarchique. Pour compléter cette vue, la partie basse de l'écran contient une vue globale et un cartouche précisant le thème et la position de la vue locale [Begeman \& Conklin 88].

\section{Aides historiques}


Ces aides ont trait au contexte temporel et à l'aspect dynamique de la consultation. Elles répondent aux questions relatives à ce qui a déjà été vu ou à ce qui reste encore à voir.

- Les historiques (history trails) mémorisent les activités de l'utilisateur. Ils lui permettent notamment de survoler ce qu'il a déjà vu et de revenir facilement sur ses activités passées. En HyperCard, les quarante-deux dernières étapes du chemin parcouru sont enregistrées sous forme de miniatures et un clic sur n'importe laquelle d'entre elles permet de retourner $\mathrm{au}$ document sans avoir à se rappeler exactement où il est et comment on $\mathrm{y}$ accède [Goodman 88]. Le système Intermedia, lui, mémorise le chemin parcouru sous forme d'une simple liste des activités de l'utilisateur, mais offre à celui-ci une aide temporelle plus complète en mélangeant trois approches différentes : le chemin (path), la vue (map) et l'enchaînement des thèmes (scope line). Il dispose aussi de trois autres facilités importantes : la mise-à-jour dynamique de la carte de l'utilisateur, la prévisualisation des liens, et les raccourcis [Utting \& Yankelovich 89].

- Les empreintes (footprints) résultent d'un marquage du système. Elles sont automatiquement déposées dès que l'utilisateur passe sur un noeud [Nielsen 90b].

- Les repères ou signets (landmarks), contrairement aux empreintes, correspondent à des marques posés par l'utilisateur au cours de sa navigation dans l'hyper-espace. Ils lui permettent de mettre en valeur des informations importantes pour lui.

- Les indices de progression (progression cues) ne sont pas très souvent utilisés dans les systèmes de type hypertexte. Par des effets visuels, ils signalent ce que l'utilisateur a déjà vu. Ils influent sur des abstractions de plus haut niveau (vue, index ou hiérarchie) que les empreintes (noeud).

L'ensemble des aides passées en revue montre la diversité des outils proposés par les chercheurs. Toutes ont pour fonction de faciliter la navigation des utilisateurs dans l'hypertexte. Mais les outils à mettre à leur disposition dépendent des usages envisagés et des objectifs à atteindre. Néanmoins, pour optimiser le processus de recherche de diverses catégories d'utilisateurs, il est souhaitable que les outils et l'interface s'adaptent dynamiquement à leurs besoins. Pour ce faire, les techniques de l'intelligence artificielle apportent des pistes prometteuses. Les solutions à envisager ne sont sans doute pas générales, mais dépendent du type d'application et du contexte dans lequel l'hypertexte est utilisé. 


\subsection{Classification des hypertextes}

Pour classer les systèmes hypertextes, diverses taxonomies ont été proposées. On peut en citer brièvement quelques unes.

- Conklin répartit les systèmes en quatre grandes classes [Conklin 87] :

(1) macro-littéraires (pour des corpus importants),

(2) exploration de problèmes,

(3) consultation libre ("browsing"),

(4) usage général.

- Halasz prend en compte trois critères permettant de préciser les caractéristiques des différents systèmes [Halasz 88] :

(1) le nombre d'utilisateurs et la quantité d'information gérée,

(2) l'orientation vers la consultation ou la création,

(3) le domaine d'application s'il est spécifique ou général.

- Leggett, Schnase et Kacmar proposent une classification orientée vers les applications en cinq catégories, qui recoupe partiellement celle de Conklin [Leggett \& al. 90] :

(1) littéraires,

(2) structurels,

(3) hypertextes de présentation,

(4) hypertextes de travail en collaboration,

(5) hypertextes d'exploration.

Nénamoins, comme le remarquent Laufer et Scavetta, ces classifications convainquent d'autant moins que leur précision (relative) les éloigne de l'esprit même de l'hypertexte [Laufer \& Scavetta 92]. De plus, aucun système n'est réellement limité à une catégorie particulière d'applications. Deux autres classifications, contrairement aux précédentes, peuvent nous permettre de mieux appréhender les usages des hypertextes :

- Duffy et Knuth proposent une taxonomie basée sur les types d'usage, plusieurs usages pouvant être possibles sur un même système [Duffy \& Knuth 90] :

(1) explorer un réseau d'informations important,

(2) accéder précisément à des noyaux d'information,

(3) opérer (par exemple : annoter, extraire...) sur un réseau d'informations,

(4) construire un réseau d'informations. 
- Nelson et Palumbo pensent que tous les systèmes tendent à refléter des caractéristiques d'une des approches suivantes [Nelson \& Palumbo 92] :

(1) présentation de connaissances,

(2) représentation de connaissances,

(3) construction de connaissances.

Ces deux dernières classifications se recoupent dans la mesure où elles rendent compte des possibilités croissantes offertes pour les activités de l'utilisateur et les manipulations des connaissances. Mais l'absence d'une classification consensuelle dans la communauté hypertexte montre une nouvelle fois la difficulté de cerner le concept même d'hypertexte. Par rapport à ces différentes classifications, les applications éducatives sont transversales. Néanmoins, les deux dernières propositions fondées sur les usages ou le rapport aux connaissances, peuvent nous éclairer sur les activités éducatives permises ou engendrées par les hypertextes.

\section{Hypertexte et éducation}

Les applications éducatives de l'informatique recouvrent un ensemble très vaste d'activités souvent complexes. De même, les usages éducatifs des hypertextes ou du concept d'hypertexte sont nombreux et diversifiés. Mais, en l'absence d'une définition communément admise d'un hypertexte, il est difficile de préciser de manière satisfaisante ce que pourrait être un hypertexte éducatif. Mise à part, quelques applications pionnières comme Intermedia [Garrett 86, Meyrowitz 86], l'usage éducatif est tributaire des systèmes actuellement disponibles. Ceci renforce une vision limitative de l'hypertexte, assez éloignée de ses origines et de ses problématiques de recherche. En particulier, beaucoup de systèmes séparent fortement le mode auteur du mode lecteur, ce qui est consistant vis-à-vis des développements de l'Enseignement Assisté par Ordinateur (EAO), et des contraintes éditoriales et commerciales, mais peu conforme à l'esprit même de l'hypertexte. Notre propos consiste à souligner la diversité des usages possibles et non à restreindre cette présentation à une classe d'usage particulier.

Dans la suite, nous allons tout d'abord préciser quelques caractéristiques des hypertextes éducatifs, proposer une classification basée sur les types d'usage, et enfin revenir sur les problèmes de la navigation dans un contexte éducatif.

\subsection{Quelques caractéristiques des hypertextes éducatifs}


Les hypertextes sont avant tout des systèmes de gestion de l'information, conçus pour aider l'homme dans ses activités cognitives, et pas des systèmes de formation au sens où peuvent l'être les «tuteurs intelligents». Il y a bien deux aspects complémentaires dans le concept d'hypertexte : (1) la représentation et la manipulation des connaissances selon un processus associatif et (2) un système fortement interactif, pouvant inclure des possibilités d'«immersion» dans des environnements de réalité virtuelle. La définition de l'hypertexte que nous avons donnée précédemment (Cf. fin du §2.1) a l'avantage de mettre en évidence quatre types d'activités : navigation, manipulation, communication et coopération. Dans les usages éducatifs, ses activités ne sont pas toujours présentes en même temps et peuvent prendre plusieurs formes. Les activités de manipulation, par exemple, peuvent être très diverses : création et/ou modification des ressources, création et/ou modification des liens, annotations, extractions...

Par ailleurs, divers logiciels ou applications utilisés dans ce contexte, partagent des fonctionnalités communes avec les hypertextes, sans pour autant pouvoir être considérés comme des hypertextes. Ainsi, les logiciels avec des interfaces utilisant des systèmes de pointage sur des zones d'écran ne sont pas des hypertextes ; ce sont plutôt des systèmes basés sur la navigation. Les jeux d'aventure en sont un exemple. Ils ont suivi des évolutions similaires à celles des hypertextes, passant d'interfaces limitées au texte (l'usage d'images n'intervenant que comme illustration), à la possibilité de pointer des zones d'écran pour désigner des objets, pour manipuler des personnages, incitant à une participation plus active et favorisant la coopération de plusieurs joueurs. Dans ces jeux, contrairement aux hypertextes éducatifs, les informations nécessaires sont cachées et leur accès est volontairement rendu difficile, le but étant de les découvrir. C'est d'ailleurs souvent au joueur de créer ses propres repères et de s'orienter dans ces univers souvent très complexes. La vidéo interactive, elle non plus, ne peut être assimilée à de l'hypertexte car comme son nom l'indique ou ne l'indique pas, l'interaction est souvent minimale, les capacités d'intervention offertes étant la plupart du temps trop limitées. La navigation consiste à choisir non seulement ce que l'on va voir, mais aussi comment on va le voir.

\subsection{Usages éducatifs des hypertextes}

Les hypertextes peuvent être vus comme des systèmes d'information conçus pour rendre l'information plus facile à retrouver/créer, ou comme des réseaux de documents interconnectés qui peuvent constituer des composantes de systèmes d'instruction.

Pour classifier les usages éducatifs de l'hypertexte, on peut s'appuyer sur trois critères : 
(1) les activités de l'apprenant, reprenant en cela la classification de Duffy et Knuth et la définition de Maurer ;

(2) le type d'aide ou de contrôle sur ces activités ;

(3) les formes d'apprentissage visées par la navigation ou l'exploration, par la résolution de problèmes et par la constuction ou la production d'objets informatiques.

En première approximation, la prise en compte de ces différents critères croise la classification de Taylor [Taylor 80] concernant l'usage des ordinateurs dans l'éducation. Dans cette classification classique, d'ailleurs peut-être trop classique ${ }^{3}$, Taylor distingue trois rôles différents pour la machine ; l'ordinateur est vu comme :

- Outil (Tool), c'est le cas du traitement de texte, des outils de calcul, des programmes d'analyse statistique...

- Enseignant (Tutor), c'est le cas classique de l'ordinateur tuteur, ayant pour origine l'enseignement programmé puis l'Enseignement Assisté par Ordinateur (EAO) ;

- Enseigné (Tutee), où l'apprenant enseigne en quelque sorte à la machine. C'est le cas de l'approche Logo.

Un quatrième rôle peut être ajouté, c'est celui du Jeu (Toy). Taylor l'avait signalé sans toutefois l'inclure, considérant qu'il pouvait s'intégrer dans les trois rôles déjà définis. Une classification similaire a été proposée par Romiszowski pour les applications éducatives de l'hypertexte [Romiszowski 90]. La superposition de ce point de vue lié au statut de la machine au regard du type d'activités de l'apprenant, donne une image assez complète de l'usage des hypertextes en éducation (Cf. Figure 2).

3 Nous reviendrons sur ce point un peu plus loin 


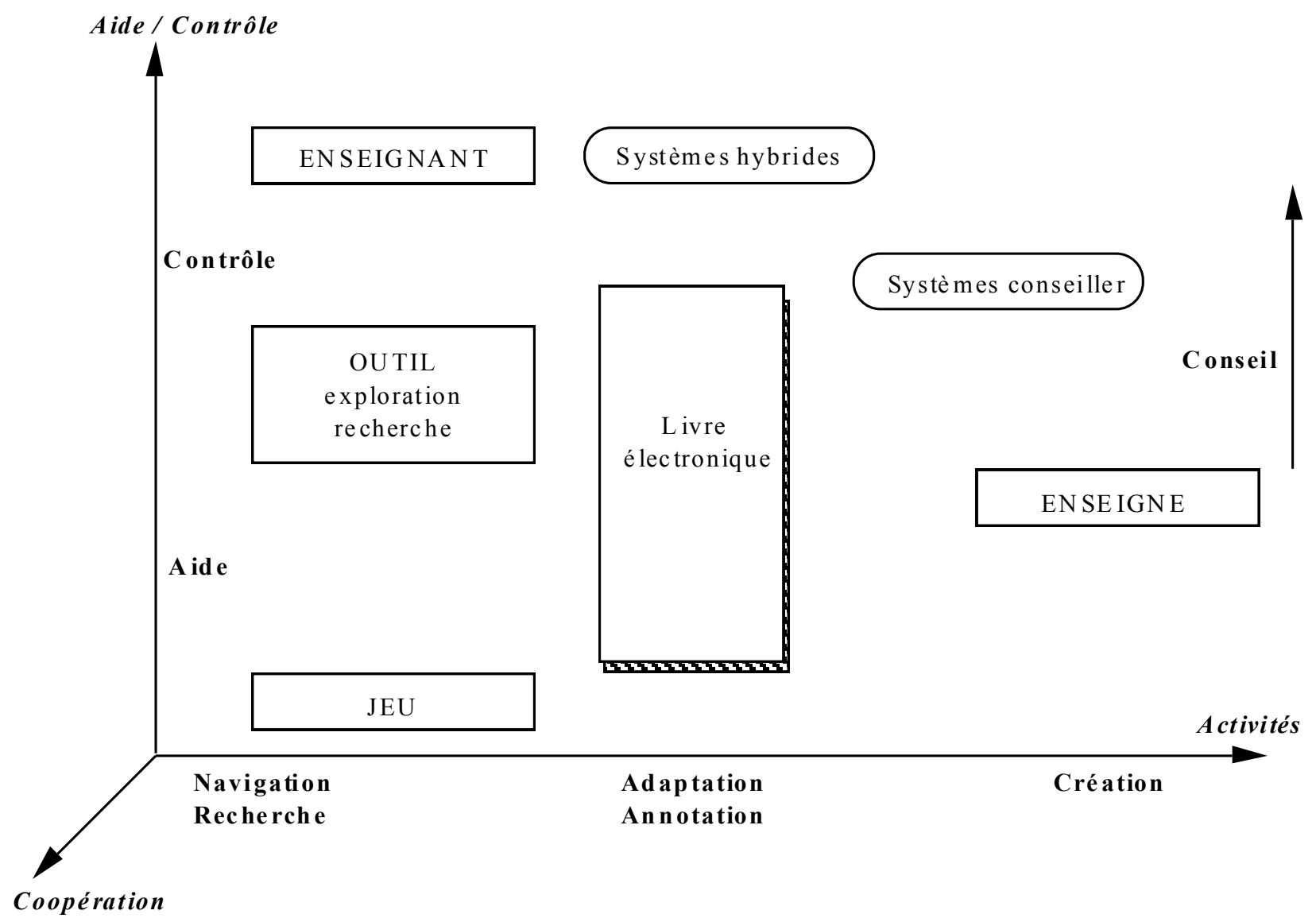

Figure 2: usages éducatifs des hypertextes

\section{L'hypertexte, en tant qu'outil}

Il est vu ici comme un sytème dans lequel on peut puiser des informations. Le prototype est l'encyclopédie électronique. En accord avec Duffy et Knuth, deux modes de consultation très différents peuvent être effectués [Duffy \& Knuth 90] : l'exploration libre de grandes bases de données et la recherche d'informations spécifiques. Un même hypertexte peut bien sûr permettre ces deux types d'exploration. Ces activités s'insèrent naturellement dans des situations éducatives plus générales.

La recherche d'informations comme méthode pédagogique a longtemps été négligée, sans doute en raison des difficultés d'accès aux sources d'informations, mais prend désormais de plus en plus d'importance. Le Meur valorise les stratégies d'apprentissage engageant l'apprenant dans «une démarche d'évaluation du manque (ce qu'il veut savoir), d'identification des ressources potentielles et de leur organisation, puis dans une stratégie d'acquisition» [Le Meur 90].

L'exploration libre dans des bases d'information, privilégiant un mode d'apprentissage par la découverte (ou par «vagabondage») soulève deux problèmes. L'interactivité, même forte, ne 
suffit pas. L'engagement de l'apprenant a plus de chances d'être réel si une tâche lui incombe, c'est-à-dire si son exploration est finalisée, comme par exemple dans la production d'un document ou dans la résolution d'un problème. L'aspect motivation est essentiel. En outre, on peut mettre en doute la capacité d'un néophyte à trouver et à comprendre quelque chose dans un domaine qu'il ne connaît pas encore. Le matériel brut, suffisant pour les experts, est inadéquat pour les néophytes. Les bases d'informations, pour être utiles en formation, doivent être conçues dans ce but et l'exploration, pour être productive, doit non seulement s'appuyer sur des chemins prédéfinis (trails), mais aussi être assistée par des «guides».

\section{L'hypertexte, en tant qu'enseignant}

Dans cette vision, l'hypertexte proprement dit doit être complété par diverses fonctionnalités que l'on trouve dans les logiciels d'enseignement. On peut adopter deux approches pour la conception de ces systèmes hybrides :

1. Ajouter des fonctionnalités hypertextes à un logiciel d'enseignement. L'hypertexte est vu alors comme une ressource complémentaire, ayant un rôle de nature essentiellement documentaire (présentation du contenu, messages d'aide et messages d'erreurs...). On peut par exemple utiliser un hypertexte fonctionnant en mode résident (présent en mémoire en même temps qu'une autre application), avec des modes d'accès contrôlés, comme dans Seve [Bruillard 91a].

2. Ajouter des fonctionnalités liées d'enseignement (guidage, exercices, évaluation...) à un système extensible, bâti sur la navigation ou à un générateur hypertexte, comme dans HyperCard [Beltran 91, Beltran 93].

Dans le premier cas, il s'agit d'une évolution des langages auteurs par ajout de ressources hypertextes ; dans le second, on transforme des générateurs hypertextes en des langages auteurs. Par rapport aux logiciels d'enseignement traditionnels, il s'agit de rendre, au moins partiellement, le contrôle à l'apprenant dans un environnement riche bénéficiant de ressources multi-médias et d'une organisation hypertextuelle. Les liaisons possibles entre les fonctionnalités hypertextes et les activités proposées sont déterminantes (en particulier, le contrôle éventuel d'accès contextuels). Dans cette perspective, E. Forte propose une classification distinguant hypertexte de documentation, hypertexte d'aide en ligne et hypertexte pédagogique, ce dernier remplissant la fonction de base d'aide théorique du didacticiel auquel il est couplé [Forte \& al. 93]. 


\section{L'hypertexte, en tant qu'enseigné}

L'hypertexte est ici vu comme un générateur que l'on utilise pour créer, individuellement ou en groupe, un réseau structuré d'informations. Dans ce contexte, le ou les apprenants ont le rôle d'auteur, auquel il est donc nécessaire de leur fournir des outils de construction adaptés. L'hypertexte s'apparente dans ce cas-là à un micro-monde [Bruillard 91b].

L'aspect coopération s'accompagne souvent d'une perspective de construction sociale du savoir, conduisant à privilégier les travaux collectifs aux travaux individuels. L'hypertexte rend alors externe la connaissance personnelle et structure le discours public. Dans le projet CSILE (Computer-Supported Intentional Learning Environments), un système hypertexte est construit autour d'une base de données générée par les apprenants [Scardmalia 91, Barrett 92].

Entre l'hypertexte enseignant et l'hypertexte enseigné se situent les environnements de production ou de communication à base d'hypertextes, individuels ou coopératifs [Derycke 91]. Les ressources hypertextes sur un domaine sont exploitées (extraites, complétées, reliées...) en vue d'une production personnelle ou collective et le processus de production peut être guidé par un système conseiller comme dans LOUTI [Paquette 91]. On peut mentionner dans cette catégorie les systèmes de support d'argumentation basés sur l'hypertexte tels g-IBIS [Conklin 87] ou SEPIA [Streitz et al. 92].

\section{L'hypertexte, en tant que jeu}

De ce point de vue, l'hypertexte est plus proche des systèmes à base de navigation. C'est le cas déjà signalé des jeux d'aventure. L'introduction, dans le déroulement du jeu, de phases de questions-réponses ajoute des fonctionnalités enseignantes. C'est le cas, par exemple dans les "Anneaux de Beltane" [Beltane 92]. Néanmoins, ces ajouts ne doivent pas être omniprésents sous peine d'anihiler l'aspect ludique 4 .

$\mathrm{Au}$ croisement de ces différents rôles, le livre électronique fournit un environnement intégré où les aspects «livres sur écran» sont complétés par diverses ressources (exercices, simulations, etc.) pour l'apprentissage autonome. Le concept de «livre carnet», livre dynamique et interactif, de type Dynabook [Kay \& Goldberg 77, Goldberg 79], offre des possibilités d'annotation et de réorganisation, et donc de manipulation des connaissances intéressantes.

4 Les générateurs EAO se parent des attributs des jeux d'aventure. Ils utilisent des thèmes évocateurs liés au conte, au parcours initiatique et correspondent davantage à un usage à domicile qu'à un usage scolaire. 
Du point de vue de l'enseignant, l'hypertexte peut constituer une aide appréciable, comme ressource de présentation collective ou comme assistance à la production de documents. Dans une perspective de construction d'hypertextes par les apprenants, l'enseignant devrait pouvoir jouer le rôle d'ouvreur de pistes (trail blazer) [Bush 45] pour les apprenants plutôt que celui de collecteur de ressources.

\section{Au-delà de la classification de Taylor}

La classification de Taylor n'est peut-être plus opératoire mais elle reste indicative d'un certain mode d'appropriation de l'hypertexte par les communautés éducatives. La distinction entre "Outil"et "Enseigné" semble de moins en moins pertinente. Elle suppose un écart entre les connaissances elles-même et les différentes façons de les manipuler et tient peu compte des aspects sociaux de la construction du savoir. L'ambition de l'hypertexte se situe bien au-delà et tend à modifier la notion même de document dans sa production, sa gestion et sa manipulation. Il s'agit d'en faire un outil cognitif pédagogique [Kommers \& al. 92, Mayes 93]. Nous ne détaillerons pas cette approche qui semble prometteuse en nous contentant de remarquer que l'on retrouve l'idée initiale développée par Engelbart basée sur l'hypothèse que 'l'habileté d'un homme à contrôler la manipulation externe de symboles en temps réel, en réponse aux besoins minute par minute de son processus de pensée, a un effet profond sur la structure globale des concepts et des méthodes utilisés dans son activité intellectuelle" [Engelbart 62].

\subsection{Navigation dans le contexte éducatif}

Dans le contexte éducatif, il ne faut pas négliger le fait que la maîtrise d'un système hypertexte réclame des aptitudes nouvelles que les utilisateurs n'ont pas encore développées : aptitude pour la navigation, intégration d'informations, structuration du domaine, etc. Ceci nécessite de nouvelles stratégies dont l'acquisition peut, d'ailleurs, constituer en soi un objectif pédagogique des hypertextes.

Une étude en contexte scolaire de J-F. Rouet montre que l'utilisation d'un système hypertexte demande la mise en oeuvre de conduites de lecture spécifiques [Rouet 91]. Les élèves ne disposent pas d'emblée de stratégies optimales. Ce constat amène à poser la question des méthodes d'apprentissage à favoriser pour familiariser les sujets à la situation

hypertextuelle. Ce chercheur suggère par ailleurs qu'un aménagement de l'interface, par l'isolement spatio-temporel des différents types d'information, par exemple, pourrait favoriser l'orientation locale des processus de traitement [Rouet 90]. A. Le Meur pense que 
ce nouveau media doit s'inscrire que dans la continuité des supports classiques (le livre et la banque de données) et de leur mode de représentation et d'organisation de connaissances [Le Meur 90].

Sur le plan éducatif, certains phénomènes de désorientation constituent un mal nécessaire, voire même un moteur pour l'apprentissage dans la mesure où les apprenants doivent se poser des questions et tenter d'y répondre. Cependant, les problèmes de navigation peuvent être révélateurs de profondes difficultés. En effet, c'est au lecteur qu'incombe la responsabilité de donner du sens à ses pérégrinations dans l'enchevêtrement des liens de l'hypertexte. L'acte d'interprétation est essentiel. Il y a un risque certain que l'utilisateur se contente de rester à la surface et n'intègre rien de ce qu'il parcourt. A l'opposé, des chemins trop fortement balisés suppriment l'intérêt de l'hypertexte. On oscille ainsi entre une liberté totale et un parcours fortement guidé, voire fermé. Face à un tel dilemne, un tuteur ou un guide expérimenté peuvent peut-être nécessaires et les techniques d'intelligence artificielle conduirent à des solutions intermédiaires.

Une autre attitude consiste à adopter une position plus radicale, en considérant que le fait de surmonter la désorientation incombe à l'utilisateur. C'est un obstacle qu'il doit franchir pour accéder à une bonne connaissance du domaine présenté. Si les aides conventionnelles à la navigation sont nécessaires pour que les lecteurs trouvent leur chemin dans le réseau, elles sont de peu de valeur dans la navigation plus fondamentale dans l'espace conceptuel qui concerne l'apprentissage. Se perdre ne dépend alors pas de la possibilité de trouver une carte (au sens de "map"), mais de savoir si l'apprenant peut en créer une [Mayes 93]. Cette complexité est dans la nature même de l'hypertexte, il faut travailler avec, et non la traiter comme un défaut à surmonter [Slatin 88]. Confier au lecteur la responsabilité d'accéder à l'information, de trouver des pistes et dériver du sens à partir des informations requiert de sa part un investissement intellectuel plus important. Mais cette augmentation de charge cognitive apparaît consistente avec les conceptions constructivistes de l'apprentissage et semble même nécessaire [Jonassen \& Grabinger 90].

Néanmoins, certains outils d'aide à la navigation, comme ceux décrits auparavant (Cf. §2.3) peuvent apporter une assistance non négligeable en aidant l'apprenant à saisir l'étendue du domaine ou sa position actuelle dans la base d'informations. C'est notamment le cas des outils qui montrent les objectifs à atteindre, qui suggèrent un chemin dans la base, qui donnent des indices sur la progression de l'apprenant et d'une certaine manière qui incitent à poursuivre l'exploration et l'apprentissage. Ainsi, les aides ponctuelles et structurelles doivent, par exemple, mettre en relief le contexte courant pour que l'apprenant ne soit pas 
obligé de se rappeler de ce qu'il peut explorer, ni de où il est dans le réseau d'informations. De plus, une identification claire du contexte peut faciliter l'organisation de la mémoire à long terme.

Une adaptation automatique de l'interface au contexte courant et à l'historique de la consultation peut aussi s'avérer très utile. Les aides structurelles devraient évoluer au fur et à mesure de la progression de l'apprenant : par exemple, une vue grand angle devrait faire apparaître de nouvelles zones à explorer. De même, l'historique peut être représenté au moyen d'empreintes de différents niveaux de gris : les empreintes étant plus ou moins foncées en fonction du degré d'exploration de la hiérarchie. Si l'apprenant ne fait que passer dans un thème, l'empreinte reste de couleur claire ; si au contraire, il explore en profondeur le thème, celle-ci devient plus foncée, et vire au noir si l'ensemble du thème a été exploré. Des tests réalisés en ajoutant ce type de nuance montrent que le taux d'exploration de la base augmente de manière significative [Leblanc 92, Dufresne 91]. Ainsi, des empreintes intelligentes exprimant le degré d'achevement, en suivant une cohérence plus cognitive que physique, peuvent permettre de s'assurer que l'apprenant a consulté l'information pertinente, ceci sans contrainte particulière.

Une autre manière d'aider l'apprenant à contrôler la complexité de l'hyper-espace est de l'autoriser à restructurer l'information. Dans cette optique, l'afficheur graphique de MultiWorks permet à l'apprenant de déplacer physiquement les noeuds sur l'écran, le système se chargeant alors de recalculer et d'afficher automatiquement la vue globale correspondante [Morreto 90].

Enfin, pour s'assurer que l'apprenant non seulement voit mais acquiert certaines connaissances, un niveau de contrôle peut s'avérer nécessaire [Jonassen \& Mandl 90]. L'accès à l'information peut être bloqué, imposé ou émaillé de conseils supplémentaires et le système peut ainsi partiellement s'adapter au niveau de connaissances de l'apprenant. Mais ce contrôle doit être bien intégré à l'environnement pour que l'apprenant ne soit pas surpris ; il doit soit être négocié avec l'apprenant soit complétement caché.

Ainsi, dans un contexte éducatif, la navigation ne peut se réduire à une simple exploration. L'apprenant doit certes être libre d'explorer selon ses envies, ses préférences et ses besoins, mais il doit pouvoir s'orienter, s'organiser ou même planifier son travail. L'hypertexte doit pour cela lui founir les outils adéquats ou être à même de lui proposer un guidage effectif.

\section{Conclusion}


Les usages éducatifs de ce concept mouvant qu'est l'hypertexte, sont nombreux et divers. Dans le cas particulier de l'apprentissage par navigation (learning by browsing), la conception doit prendre en compte la spécificité de cette approche. Elle nécessite ainsi la mise en forme des ressources et l'ajout d'une expertise. Celle-ci a pour objet de créer des chemins pertinents pour différentes catégories d'utilisateurs. Cette expertise est tout aussi délicate à introduire que dans les systèmes d'Enseignement (Intelligemment ou non) Assisté par Ordinateur. Qu'il s'agisse d'un parcours linéaire et prédéfini comme dans l'enseignement programmé, d'un chemin quasi-linéaire, contrôlé par le système ou partiellement choisi par l'utilisateur comme en EAO et en EIAO, ou encore d'un chemin entièrement déterminé par l'utilisateur comme dans les hypertextes, il n'en reste pas moins vrai que tous nécessitent un découpage des ressources et une possibilité de créer des chemins cohérents dans ces ressources. Ce dernier aspect constitue une réelle difficulté d'un point de vue de l'expertise didactique.

Comme nous l'avons dit précedemment, ce domaine de recherche est en pleine expansion, et les applications éducatives commencent à se multiplier. Il semble intéressant d'étudier d'une de manière plus précise les usages réels qui se développent avec la mise à disposition de ces nouveaux outils et de voir comment les différents domaines disciplinaires s'approprient cette technique. Ceux-ci peuvent, en effet, avoir des approches très différentes dues à leur spécificité. Cette étude des usages est capitale, tant sur le plan de l'évaluation que de la conception d'environnements éducatifs à base d'hypertexte.

\section{REFERENCES}

[Akscyn \& al. 88] Akscyn R., McCracken D. \& Yoder E., "KMS, a Distributed Hypermedia System for Managing Knowledge in Organizations", Communications of ACM, Vol. 31, nº July 1988, pp. 820-835.

[Balpe 91] Balpe J.P., “Hyperdocuments, hypertextes, hypermédias”, Eyrolles, 1990, 200p.

[Barrett 92] Barrett E., "Sociomedia: Multimedia, Hypermedia and the Social Construction of Knowledge”, MIT Press, 1992, 581p.

[Begeman \& Conklin 88] Begeman M. \& Conklin J., "The Right Tool for the Job", BYTE, October 1988, pp.255-266.

[Beltane 92] "Les anneaux de Beltane - pour qu'apprendre devienne une aventure", logiciel sous HyperCard, 1992. 
[Beltran 91] Beltran T., "Une architecture pour le guidage de l'apprenant dans un système Hypermédia éducatif', in Hypermédias et Apprentissage (B. de La Passardière \& G.-L Baron, eds), INRP-MASI, 1991, pp.95-110.

[Beltran 93] Beltran T., "Hypermédias éducatifs: de la théorie à la pratique" in Hypermédias et Apprentissage '93 (G.-L. Baron, J.Baudé \& B. de La Passardière, eds), INRP, 1993, pp.169-180.

[Berk \& Devlin 91] Berk E., Devlin J. (eds.), "Hypertext / Hypermedia Handbook", McGraw-Hill, 1991, 583p.

[Bruillard 91a] Bruillard E., "Mathématiques et EIAO: une vision hypertexte des environnements d'apprentissage", Thèse de l'Université du Maine, Le Mans, Février 1991.

[Bruillard 91b] Bruillard E., "Some new Logo microworlds and their impact in the classroom - The future: Hypertext and Microworlds", Proceedings of the third European Logo Conference, A.S.I. Parma, 1991, pp.493-506.

[Bush 45] Bush V., “As We May Think”, Atlantic Monthly, Vol.176, n¹, 1945, pp.641649.

[Conklin 87] Conklin J., "Hypertext : an Introduction and Survey", IEEE Computer, September 1987, pp.17-41.

[Delisle \& Schwartz 86] Delisle N. \& Schwartz M., "Neptune : a Hypertext System for CAD Applications", ACM Transactions, 1986.

[Derycke 91] Derycke A., "Hypermédia et apprentisssage coopératif”, in Hypermédias et Apprentissage (B. de La Passardière \& G.-L Baron, eds), INRP-MASI, 1991, pp.77-88.

[Duffy \& Knuth 90] Duffy T.M., Knuth R.A., "Hypermedia and Instruction: Where is the Match?" in [Jonassen \& Mandl 90], pp.199-226.

[Dufresne 91] Dufresne A., "Ergonomie cognitive, hypermédias et apprentissages", in Hypermédias et Apprentisage (B. de La Passardière \& G.-L Baron, eds), INRP-MASI, 1991

[Engelbart 62] Engelbart D. C., "Program on Human Effectiveness", Stanford Research Institute, 1962, in [Nyce \& Kahn 91], pp.237-244. 
[Engelbart \& English 68] Engelbart D. C. and English W. K., "A Research Center for Augmenting Humain Intellect", AFIPS Conf. Proc., Vol. 33, Part 1, Thompson Books, Wash., 1968

[Forte \& al. 93] Forte E.N., Herzog J.M.\& Wentland M.C., "Identification de concepts et parcours oriené dans un hypertexte pédagogique" in Journées EIAO-ENS de Cachan (M.Baron, R.Gras\& J.F.Nicaud, eds), Eyrolles, 1993, pp.169-180.

[Foss 88] Foss C.L., "Effective Browsing in hypertext systems", Proceedings of RIAO 88, Cambridge, MA-MIT, 1988.

[Furnas 86] Furnas G., "Generalized fisheye views", in CHI'86 Human Factors in Computing Systems Proc., ACM, Boston, April 13-19, New York, 1986, pp. 16-23.

[Garrett 86] Garrett N. L., Smith K. E. \& Meyrowitz N., "Intermedia : Issues, Strategies, and Tactics In the Design of a Hypermedia Document System", in Computer-Supported Cooperative Work Conf. Proc., MCC Software Technology Program, Austin, Texas, 1986.

[Garg 88] Garg P., "Abstraction Mechanisms in Hypertext", Communications of ACM, Vol. 31, n 7, July 1988, pp. 862-870.

[Goldberg 79] Goldberg A., "Educational uses of a dynabook", Computers \& Education, Vol.3,1979, pp.247-266.

[Goodman 88] Goodman D., "The Complete HyperCard Handbook, Second Edition", Bantam Books, October 1988.

[Halasz 88] Halasz F., "Reflections on NoteCards : Seven Issues for the Next Generation of Hypermedia Systems", Communications of ACM, Vol. 31, n 7, July 1988, pp. 836 - 852.

[Hill 86] Hill B., "Guide : Hypertext for the Macintosh", Owl International Inc., Bellevue, Wash., 1986, pp.25-39.

[Jonassen \& Grabinger 90] Jonassen D.H., Grabinger R.S., "Problems and Issues in Designing Hypertext/Hypermedia for Learning”, in [Jonassen \& Mandl 90], pp.3-25.

[Jonassen \& Mandl 90] Jonassen D. \& Mandl H., "Designing Hypermedia for Learning", Nato, Serie F, Vol. 67

[Jonassen 93] Jonassen D., "Applications of Hypertext Technologies for Higher Education", Journal of Computing in Higher Education, vol 4 (2), 1993, pp. 12-42. 
[Kay \& Goldberg 77] Kay A., Goldberg A., "Personal dynamic media", Computer, Mars 1977, pp. 31-41.

[Kommers \& al. 92] Kommers P., Jonassen D. \& Mayes J.T. (eds.), “Cognitive Tools for learning", Heidelberg, FRG, Springer-Verlag, 1990.

[Laufer \&Scavetta 92] Laufer R., Scavetta D., "Texte, Hypertexte, Hypermédia”, Que saisje? n²629, PUF, 1992, 123p.

[Leblanc 92] Leblanc V., "Influence de l'organisation graphique et des indices de progression sur la navigation dans un environnement hypertexte". Mémoire de maîtrise, Université de Montréal, 1992

[Legett \& al.] Leggett J.J., Schnase J.L. \& Kacmar C.J., "Hypertext for Learning" in [Jonassen \& Mandl 90], pp.27-38.

[Le Meur 90] LeMeur A., "Hypertext and more. ARGOS: a new approach to learning by seeking", Applica 90, Lille, Septembre 1990.

[Mayes 93] Mayes T., "Hypermédias et outils cognitifs" in Hypermédias et Apprentissage 2 (G.-L. Baron, J.Baudé, B. de La Passardière, eds), INRP, 1993, pp.39-47.

[Maurer 92] Maurer H., “Why Hypermedia Systems are important?”, in proceedings ICCAL 92, (I.Tomek, ed.), Lecture notes n602, Springer Verlag, 1992, pp.1-15.

[Meyrowitz 86] Meyrowitz N., "Intermedia : the Architecture and Construction of an Object Oriented Hypermedia System and Applications Framework", OOPSLA'86 Proc., September 1986.

[Meyrowitz 89] Meyrowitz N., "Hypertext - Does It Reduce Cholesterol Too?" Keynote address for the Hypertext'89 Conference in Pittsburg, PA, Novembre 1989, in [Nyce \& Kahn 91], pp. 285-318.

[Meyrowitz 90] Meyrowitz N.,'Responsive Documents - The Call of the 90s", Préface, TSI vol.9, $\mathrm{n}^{\circ} 6,1990$.

[Morreto 90] Morreto R., "Multiworks - a MULTImedia Integrated WORKStation", in Esprit'90 Conf. Proc., Brussels, Nov 12-15, Kluwer Academic Press, 1990, pp. 654-668.

[Nanard 90] Nanard M., "Présentation", TSI vol.9, n6, 1990, p.493. 
[Nielsen 90a] Nielsen J., "Hypertext and Hypermedia”, Academic Press, San Diego, 1990, $263 p$.

[Nielsen 90b] Nielsen J., "The Art of Navigating through Hypertext", Communications of ACM, Vol. 33, n 3, March 1990.

[Nelson \& Palumbo 92] Nelson W.A., Palumbo D.B., "Learning, Instruction and Hypermedia", Jl. of Educational Multimedia and Hypermedia, 1992, vol 1, n³, pp.287299.

[Nyce \& Kahn 91] Nyce J.M., Kahn P.(eds), "From Memex to Hypertext, Vannevar Bush and the Mind's Machine", Academic Press, 1991, 367p.

[Paquette 91] Paquette G., "Métaconnaissances dans les environnements d'apprentissage", Thèse de l’Université du Maine, Le Mans, Octobre 1991.

[Pasquier-Boltuck \& al. 89] Pasquier-Boltuck J., Collaud G., Monnard J., “Conception et programmation par objets d'un système interactif de création et de consultation de livres électroniques", Bigre 63-64, Mai 1989, pp. 7-17.

[Rouet 90] Rouet J.F., "Lecture et Orientation Sémantique dans les documents nonlinéaires", Applica 90, Lille, septembre 1990.

[Rouet 92] Rouet J.F., "Apprendre à lire un hypertexte, une étude expérimentale”, Cahiers de Linguistique sociale, 1992.

[Scardmalia 91] Scardmalia M., "An architecture for the social construction of knowledge", in Proceedings of the International Conference on the Learning Sciences, AACE (L.Birnbaum, ed.), 1991, p.397.

[Shneiderman \& Kearsley 90] Shneiderman B. \& Kearsley G., "HYPERTEXT HANDS-ON : an Introduction to a New Way of Organizing and Accessing Informations", AddisonWesley Publishing Company, 1990.

[Slatin 88] Slatin J.M., "Hypertext and the teaching of Writing", in "Text, Context and Hypertext”, MIT Press (E.Barrett, ed.), 1988, pp.111-129.

[Streitz 92] Streitz N.A., Haake J., Hanneman J., "Sepia a cooperative Hypermedia Authoring Environment", Proceedings of the 4TH ACM Conference on Hypertext, ECHT'92, Milan. 
[Rheingold 93] Rheingold H., “La réalité virtuelle”, Dunod, Paris, 1993, 413p.

[Romiszowski 90] Romiszowski A.J., "The Hypertext/Hypermedia Solution - But What Exactly is the Problem" in [Jonassen \& Mandl 90], pp.321-354.

[Savoy 88] Savoy J., “EBOOK3 Le livre électronique”, TSI, Vol.7, n5, 1988, pp.437-450.

[Taylor 80] Taylor R.P.(ed.), “The Computer in the School: Tutor, Tool, Tutee”, New York: Teachers College Press, 1980.

[Trigg 83] Trigg R. H., "A Network-based Approach to Text Handling for the On-line Scientific Community", PhD Thesis, University of Maryland, 1983.

[Utting \& Yankelovich 89] Utting K. \& Yankelovich N., "Context and Orientation in Hypermedia Net-works", ACM Transactions on Information Systems, Vol. 7, $\mathrm{n}^{\circ}$ 1, January 1989, pp. 58-84.

[Vatonne 90] Daniel-Vatonne M.C., "Hypertextes: des prinicpes communs et des variations", TSI Vol.9, n6, 1990, pp. 475-492. 